\title{
IMPACTO DE LA APLICACIÓN DE LOS CRITERIOS DE LA ASOCIACIÓN AMERICANA DE TIROIDES EN EL DIAGNÓSTICO DE HIPOTIROIDISMO EN GESTANTES DE VIGO, ESPAÑA
}

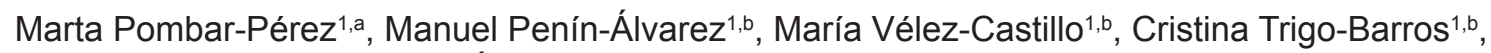 \\ Elías Álvarez-García ${ }^{1, a}$, Rosa Rodríguez-Ferro ${ }^{2, c}$
}

\section{RESUMEN}

Con el objetivo de determinar el impacto de la aplicación de los criterios de la Asociación Americana de Tiroides (ATA) en el diagnóstico de hipotiroidismo en gestantes del área sanitaria de Vigo en España, se analizó la concentración sérica de tirotropina (TSH), tiroxina libre (T4L) y anticuerpos antitiroideos, comparando la frecuencia de gestantes diagnosticadas de hipotiroidismo aplicando los criterios utilizados en el laboratorio de hormonas del Hospital Xeral y los criterios de referencia propuestos por la ATA. La asunción de dichos criterios implicaría un aumento de un $29,6 \%$ de pacientes diagnosticadas de hipotiroidismo. La aplicación de los criterios ATA tuvo una repercusión en la medición de la concentración de TSH en gestantes lo que sugiere una evaluación de los valores de referencia de TSH en función de la población y los métodos de diagnóstico locales.

Palabras clave: Hipotiroidismo; Embarazo; Enfermedades de la tiroides (fuente: DeCS BIREME).

\section{IMPACT OF THE APPLICATION OF THE AMERICAN THYROID ASSOCIATION CRITERIA ON THE DIAGNOSIS OF HYPOTHYROIDISM IN PREGNANT WOMEN IN VIGO, SPAIN}

\begin{abstract}
In order to determine the impact of the application of the American Thyrold Associations (ATA) criteria for the diagnosis of hypothyroidism in pregnant women in the health district of Vigo, Spain, concentrations of serum thyrotropin (TSH), free thyroxine (T4L) and anti-thyroid antibodies were analyzed, comparing the frequency of pregnant women diagnosed with hypothyroidism and applying the criteria used in the hormone laboratory at Xeral Hospital and referential criteria established by ATA. The application of ATA referential criteria increased by $29.6 \%$ the number of female patients diagnosed with hypothyroidism. The application of ATA guidelines had an impact on the measurement of TSH concentrations in pregnant women, which calls for an evaluation of referential values of TSH based on the population and the local diagnostic methods.
\end{abstract}

Key words: Hypothyroidism; Pregnancy; Thyroid diseases (source: MeSH NLM).

\section{INTRODUCCIÓN}

Durante la gestación, el aumento fisiológico de la síntesis de tiroxina como resultado de la actividad tirotropa de la hormona gonadotropina coriónica produce un descenso de la concentración de tirotropina (TSH), especialmente notable en el primer trimestre ${ }^{(1)}$.

Varias publicaciones han mostrado que el hipotiroidismo franco, definido como el aumento de la concentración de TSH con disminución de concentración de tiroxina libre (T4L), o como una concentración de TSH>10 $\mu \mathrm{Ul} / \mathrm{ml}$, independientemente de la $\mathrm{T} 4 \mathrm{~L}$ (2) se asocia a efectos nocivos en la gestación ${ }^{(3,4)}$ y en el desarrollo neurocognitivo fetal ${ }^{(5,6)}$. Hay consenso en la utilidad de tratar con tiroxina a este grupo de pacientes.

El hipotiroidismo subclínico, definido como el aumento de la concentración de TSH inferior a $10 \mu \mathrm{UI} / \mathrm{mL}$ con concentración de T4L normal (2) se ha asociado también a perjuicios en el embarazo ${ }^{(7)}$, y de forma más dudosa al desarrollo neurocognitivo del feto ${ }^{(8)}$. Algunos autores defienden su tratamiento en todos los casos ${ }^{(9)}$, y otros recomiendan hacerlo sólo en

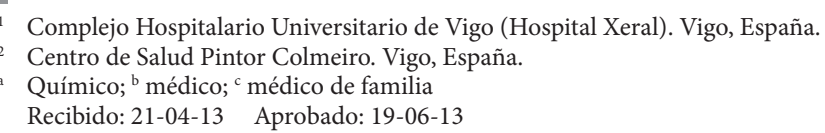

Citar como: Pombar-Pérez M, Penín-Álvarez M, Vélez-Castillo M, Trigo-Barros C, Álvarez-García E, Rodríguez-Ferro R. Impacto de la aplicación de los criterios de la Asociación Americana de Tiroides en el diagnóstico de hipotiroidismo en gestantes de Vigo, España. Rev Peru Med Exp Salud Publica. 2013;30(3):428-31. 
pacientes con concentración significativa de anticuerpos antitiroideos en suero ${ }^{(10)}$.

Para la evaluación de la función tiroidea de las gestantes se utilizan los valores de referencia propuestos por el fabricante de los reactivos de TSH y T4L. Estos valores derivan de un estudio realizado en dos ciudades de Alemania, en personas sin historia de disfunción tiroidea conocida y con concentración de TSH en el rango de 0,10-10,00 $\mu \mathrm{UI} / \mathrm{mL}$ y T4L menor de 1,72 ng/100mL. El rango de concentraciones de $\mathrm{TSH}$, expresados como los percentiles 2,5 y 97,5 son los siguientes: $0,33-4,59 \mu \mathrm{UI} / \mathrm{mL}$ en el primer trimestre; $0,35-4,10 \mu \mathrm{UI} / \mathrm{mL}$ en el segundo trimestre, y $0,21-3,15 \mu \mathrm{UI} / \mathrm{mL}$ en el tercer trimestre (11). El rango para la T4L, expresado como percentil 2,5 y 97,5 resultó: 0,94-1,52 ng/100mL en el primer trimestre; $0,75-1,32 \mathrm{ng} / 100 \mathrm{~mL}$ en el segundo trimestre y $0,65-1,21$ $\mathrm{ng} / 100 \mathrm{~mL}$ en el tercer trimestre.

Desde el año 2001, el Hospital Xeral de Vigo (España) realiza el cribado universal de disfunción tiroidea en el primer trimestre de gestación. Las gestantes que presentan valores de TSH superiores a las cifras expuestas son derivadas al Servicio de Endocrinología para su evaluación. Pese a estandarización de los inmunoanálisis para la cuantificación de TSH, los valores de referencia para población gestante, obtenidos en distintas áreas de salud de España y utilizando métodos diferentes, conducen a puntos de corte notablemente distintos a la hora de definir la hipofunción tiroidea materna ${ }^{(12)}$.

En el año 2011 la Asociación Americana de Tiroides (ATA) publicó una guía ${ }^{(2)}$ en la que recomienda el uso de los siguientes intervalos de referencia de concentración de TSH durante la gestación; primer trimestre: 0,1-2,5 $\mu \mathrm{UI} / \mathrm{mL}$; segundo trimestre: $0,2-3,0 \mu \mathrm{Ul} / \mathrm{mL}$, y tercer trimestre: 0,3-3,0 $\mu \mathrm{UI} / \mathrm{mL}$.

El objetivo de la presente investigación fue determinar la repercusión del uso de los criterios de referencia de la ATA en el diagnóstico de hipotiroidismo en gestantes del área sanitaria de Vigo, España.

\section{EL ESTUDIO}

El área sanitaria de Vigo engloba tres laboratorios de hormonas, situados en los hospitales Xeral, Meixoeiro y POVISA (Policlínico Vigo, S. A.). En el área se produjeron aproximadamente 4300 partos durante el año 2011. Se realizó un muestreo aleatorio simple de 2690 mujeres de dicha población. Se excluyó a todas aquellas mujeres cuya gestación no hubiese llegado a término. Todas las gestantes recibieron tratamiento suplementario con yodo (entre 200 y $300 \mu$ g/día) durante la gestación.

Todos los análisis de suero se realizaron en el Laboratorio de Hormonas del Hospital Xeral de Vigo. La concentración de TSH (análisis inmunométrico electroquimioluliniscente), T4L y anticuerpos antiperoxidasa (inmunoanálisis competitivo electroquimioluminiscente) se determinó en un analizador Cobas $6000^{\circledR}$ (Roche, Mannheim, Germany) y la concentración de anticuerpos antitiroglobulina (análisis inmunométrico quimioluminiscente) se midió en un analizador Inmulite $2000^{\circledR}$ (Siemens Healthcare Diagnostic, Alemania).

En 2410 (89,6\%) pacientes se analizó la concentración plasmática de TSH y de T4L en el primer trimestre de gestación. A 571 (21,2\%) gestantes se les determinó la concentración de estos parámetros en el segundo trimestre y a $392(14,6 \%)$ pacientes en el tercer trimestre. La concentración de anticuerpos antiperoxidasa tiroidea y antitiroglobulina se midió si la concentración de TSH es mayor de $4,00 \mu \mathrm{Ul} / \mathrm{mL}$, siempre que la paciente no tenga registrados en el sistema de información del laboratorio valores previos.

Se analizó la frecuencia de las gestantes que fueron clasificadas con hipotiroidismo según los criterios clásicos y los criterios ATA, y el tipo de hipotiroidismo (subclínico o clínico) que presentaron.

\section{HALLAZGOS}

La edad media de las pacientes fue $33 \pm 5$ años. La concentración media de TSH y T4L de las pacientes según cada trimestre de gestación están detalladas en la Tabla 1.

Usando como referencia los límites de TSH y T4L, actualmente usados en nuestro hospital, 301 de 2690 gestantes $(11,2 \%)$ tenían hipotiroidismo, de las cuales 58 fueron hipotiroidismo clínico y 243 subclínico, siendo este último el $80,7 \%$ de los casos de hipotiroidismo en este grupo. Al usar los criterios de referencia de la ATA, el número de casos de hipotiroidismo clínico y subclínico ascendió a 1097 gestantes $(40,8 \%$ del total de gestantes), siendo subclínico en el 93,0\% de

Tabla 1. Concentración de tirotropina (TSH) y tiroxina libre (T4L) por trimestre de gestación en gestantes de Vigo, España

\begin{tabular}{lccc}
\hline Trimestre & Gestantes & $\begin{array}{c}\text { TSH } \\
(\boldsymbol{\mu U I / m L})^{*}\end{array}$ & $\begin{array}{c}\text { T4L } \\
(\mathbf{n g} / \mathbf{1 0 0} \mathbf{~ m L})^{*}\end{array}$ \\
\hline Primer & 2410 & $2,68 \pm 1,8$ & $1,17 \pm 0,18$ \\
Segundo & 571 & $2,92 \pm 2,1$ & $1,01 \pm 0,20$ \\
Tercer & 392 & $2,90 \pm 2,3$ & $0,96 \pm 0,2$ \\
\hline
\end{tabular}

*Media \pm desviación estándar 
Tabla 2. Hipotiroidismo e hipotiroxinemia aislada según criterios actuales y de la Asociación Americana de Tiroides (ATA) en gestantes de Vigo, España

\begin{tabular}{lcclcccc}
\hline & \multicolumn{2}{c}{ Criterios actuales } & & \multicolumn{3}{c}{ Criterios ATA } & valor \\
\cline { 2 - 3 } & Gestantes & $\mathbf{( \% )}$ & & Gestantes & (\%) & p \\
\hline $\begin{array}{l}\text { Hipotiroidismo } \\
\text { clínico }\end{array}$ & 58 & $(2,2)$ & & 77 & $(2,9)$ & 0,03 \\
$\begin{array}{l}\text { Hipotiroidismo } \\
\text { subclínico }\end{array}$ & 243 & $(9,0)$ & & 1020 & $(37,9)$ & $<0,01$ \\
$\begin{array}{l}\text { Hipotiroxinemia } \\
\text { aislada }\end{array}$ & 69 & $(2,6)$ & & 37 & $(1,4)$ & 0,02 \\
\hline
\end{tabular}

${ }^{\dagger}$ Durante el primer trimestre del embarazo

los casos de hipotiroidismo. Tomando el intervalo de referencia para T4L propuesto por el fabricante, 69 gestantes $(2,6 \%)$ tenían hipotiroxinemia aislada en el primer trimestre del embarazo. Utilizando los límites recomendados por la ATA este número descendió hasta 37 gestantes $(1,4 \%$ del total) (Tabla 2$)$.

El $23,2 \%$ de estas gestantes tenía anticuerpos antiperoxidasa positivos y el 9,7\% de las gestantes tenía anticuerpos antitiroglobulina positivos.

\section{DISCUSIÓN}

Todas las gestantes pertenecientes al área sanitaria de Vigo son atendidas por servicios del Hospital Xeral. Todas estas pacientes son suplementadas con yodo a dosis de 200-300 $\mu \mathrm{g} / \mathrm{di}$ ía, descartándose de esta manera que las alteraciones de la función tiroidea observadas sean consecuencia de su déficit. La homogeneidad de la población gestante derivada de estos hechos fortalece este estudio y sus conclusiones.

En la actualidad, el Servicio de Tocología deriva a la consulta de endocrinología al $11,2 \%$ de las gestantes por diagnóstico bioquímico de hipotiroidismo. Si en lugar de aplicar los valores de referencia clásicos usados actualmente se aplicasen los nuevos criterios recomendados por la ATA, este porcentaje ascendería hasta el $40,8 \%$ de la población gestante. Esto implica un incremento del $29,6 \%$ de gestantes atendidas por el Servicio de Endocrinología. En números absolutos, en la población del área sanitaria de Vigo, con una media de 4000 partos al año, este cambio de criterios en la evaluación del hipotiroidismo supondría un aumento de 1184 pacientes en dicha consulta.

La guía de la ATA no recomienda el tratamiento sustitutivo con levotiroxina en gestantes con hipotiroxinemia aislada debido a que considera que se trata de una afección clínicamente irrelevante (2). Dicha guía sí recomienda el tratamiento de aquellas pacientes que presentan hipotiroidismo franco o bien hipotiroidismo subclínico con anticuerpos antiperoxidasa positivos. Otra repercusión del gran aumento de gestantes diagnosticadas de hipotiroidismo con los criterios de la ATA es el incremento económico del tratamiento con levotiroxina de este grupo de pacientes y, sobre todo, el riesgo del sobretratamiento de las gestantes asociado a posibles efectos adversos para la madre y el feto.

La elección del intervalo de referencia de concentración de TSH adecuado a la gestación ha generado un gran debate en los últimos años ${ }^{(13,14)}$, derivado principalmente de dos hechos. En primer lugar, las notables discrepancias observadas entre las determinaciones de TSH mediante distintos inmunoanálisis comerciales automatizados implican que los intervalos de referencia puedan llegar a ser muy diferentes según el método utilizado. Un ejemplo de esto lo encontramos en cuatro trabajos publicados en España con los valores de referencia de hormonas tiroideas de sus respectivas poblaciones en Aragón (15), Cartagena (16), Cataluña (17) y Jaén (18). En segundo lugar, la ausencia de un estudio con una población lo suficientemente amplia y adecuadamente seleccionada. Por ello, sería conveniente que cada laboratorio realizase una evaluación de los valores de referencia de TSH en función de la población y de los métodos locales.

Una de las cuestiones que preocupan a los clínicos y limitan la aplicación de los intervalos de referencia de concentración de TSH recomendados por la ATA es la posibilidad de sobretratar a estas pacientes. La administración de tiroxina a gestantes con una concentración de TSH tan baja como $2,5 \mu \mathrm{UI} / \mathrm{mL}$ podría producir un hipertiroidismo con consecuencias que desconocemos en el feto. Es posible que las dosis de tiroxina habitualmente recomendadas en el hipotiroidismo en gestantes sean excesivas si se toma como referencia los intervalos de referencia de la ATA, una cuestión que exigirá nuevos estudios.

Se concluye que existe variabilidad en la medición de la concentración de TSH mediante diferentes inmunoanálisis. Esto reafirma la conveniencia de realizar una evaluación de los valores de referencia de TSH en función de la población y los métodos de diagnósticos locales.

Contribuciones de autoría: MPP y MPA participaron en la concepción del estudio y redacción del artículo, MPP participó en la recolección, análisis e interpretación de datos. MPA y CTB participó en la revisión crítica del artículo, MVC participó en el aporte de pacientes o material de estudio, MPA y EAG participaron en la aprobación de su versión final, RRF participó en la asesoría estadística. EAG participó en la revisión crítica del artículo y en la aprobación de su versión final.

Fuente de financiamiento: autofinanciado.

Conflictos de interés: los autores declaran no tener ningún conflicto de interés. 


\section{REFERENCIAS BIBLIOGRÁFICAS}

1. Haddow JE, Knight GJ, Palomaki GE, McClain MR, Pulkkinen AJ. The reference range and within-person variability of thyroid stimulating hormone during the first and second trimesters of pregnancy. J Med Screen. 2004;11(4):170-4.

2. Stagnaro-Green A, Abalovich $M$, Alexander E, Azizi F, Mestman J, Negro $\mathrm{R}$, et al. Guidelines of the American Thyroid Association for the diagnosis and management of thyroid disease during pregnancy and postpartum. Thyroid. 2011;21(10):1081-125. doi: $10.1089 /$ thy.2011.0087.

3. LaFranchi SH, Haddow JE, Hollowell JG. Is thyroid inadequacy during gestation a risk factor for adverse pregnancy and developmental outcomes? Thyroid. 2005;15(1):60-71.

4. Stagnaro-Green A, Chen X, Bogden JD, Davies TF, Scholl TO. The thyroid and pregnancy: a novel risk factor for very preterm delivery. Thyroid. 2005;15(4):351-7.

5. Haddow JE, Palomaki GE, Allan WC, Williams JR, Knight GJ, Gagnon $\mathrm{J}$, et al. Maternal thyroid deficiency during pregnancy and subsequent neuropsychological development of the child. N Engl J Med. 1999;341(8):54955.

6. Idris I, Srinivasan R, Simm A, Page RC. Maternal hypothyroidism in early and late gestation: effects on neonatal and obstetric outcome. Clin Endocrinol (Oxf). 2005;63(5):560-5.

7. Casey BM, Dashe JS, Wells CE, McIntire DD, Byrd W, Leveno KJ, et al. Subclinical hypothyroidism and pregnancy outcomes. Obstet Gynecol. 2005;105(2):239-45.

8. De Escobar GM, Obregón MJ, del Rey FE. Maternal thyroid hormones early in pregnancy and fetal brain development. Best Pract Res Clin Endocrinol Metab. 2004; 18(2):225-48.

9. De Groot L, Abalovich M, Alexander EK, Amino N, Barbour L, Cobin RH, et al. Management of thyroid dysfunction during pregnancy and postpartum: an Endocrine Society Clinical practice guideline. J Clin Endocrinol Metab. 2012;97(8):2543-65. doi: 10.1210/ jc.2011-2803.

10. Negro R, Schwartz A, Gismondi R, Tinelli A, Mangieri T, Stagnaro-Green A. Universal screening versus case finding for detection and treatment of thyroid hormonal dysfunction during pregnancy. J Clin Endocrinol Metab. 2010;95(4):1699-707. doi: 10.1210/ jc.2009-2009. Epub 2010 Feb 3.

11. Roche Diagnostics. Reference Intervals for Children and Adults. Elecsys Thyroid Tests. Germany: Roche Diagnostics; 2009.

12. Vila L, Velasco I, González S, Morales F, Sánchez E, Lailla JM, et al. Detección de la disfunción tiroidea en la población gestante: está justificado el cribado universal. Med Clin (Barc). 2012;139(11):509.e1-509.e11. doi: 10.1016/j.medcli.2012.06.004.

13. Wartofsky L, Dickey RA. The evidence for a narrower thyrotropin reference range is compelling. J Clin Endocrinol Metab. 2005;90(9):5483-8.

14. Surks MI, Goswami G, Daniels GH. The thyrotropin reference range should remain unchanged. J Clin Endocrinol Metab. 2005;90(9):5489-96.

15. Bocos-Terraz JP, Izquierdo-Alvarez $S$, Bancalero-Flores JL, Alvarez-Lahuerta R, Aznar-Sauca A, Real-López E, et al. Thyroid hormones according to gestational age in pregnant Spanish women. BMC Res Notes. 2009;2:237. doi: 10.1186/1756-0500-2-237.

16. García de Guadiana Romualdo L, González Morales M, MartínOndarza González Mdel C, Martín García E, Martínez Uriarte J, Blázquez Abellán A, et al. Valoración de la función tiroidea durante la gestación: intervalos de referencia de tirotropina y tiroxina no unida a proteína durante el primer trimestre. Endocrinol Nutr. 2010;57(7):290-5. doi: 10.1016/j. endonu.2010.05.002.

17. Vila L, Serra-Prat M, Palomera E, Casamitjana R, de Castro A, Legaz $\mathrm{G}$, et al. Reference values for thyroid function tests in pregnant women living in Catalonia, Spain. Thyroid. 2010;20(2):221-5. doi: 10.1089/ thy.2008.0264.

18. Santiago P, Berrio M, Olmedo P, Velasco I, Sánchez B, García E, et al. Valores de referencia de hormonas tiroideas en la población de mujeres gestantes de Jaén. Endocrinol Nutr. 2011;58(2):62-7. doi: 10.1016/j.endonu.2010.12.004.

Correspondencia: Marta Pombar Pérez.

Dirección: c/ Pizarro, 22, CP: 36204 . Vigo, España

Teléfono:+34986816000

Correo electrónico:marta.pombarperez@sergas.es

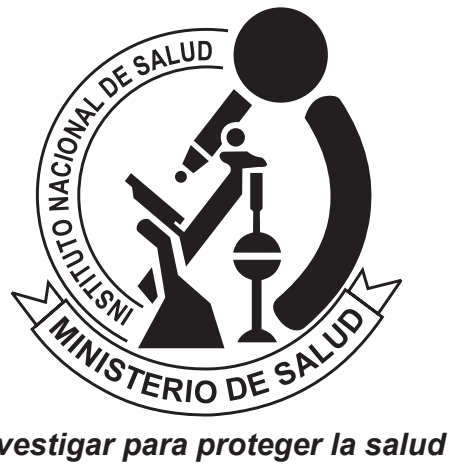

REVISTA PERUANA DE MEDICINA EXPERIMENTAL Y SALUD PÚBLICA CUMPLIENDO SUS METAS Y PROYECTÁNDOSE AL FUTURO

\section{Visite los contenidos de la revista en: www.ins.gob.pe/rpmesp}

